\title{
Klumpfuss, a putative Drosophila zinc finger transcription factor, acts to differentiate between the identities of two secondary precursor cells within one neuroblast lineage
}

\author{
Xiaohang Yang, Sami Bahri, Thomas Klein, and William Chia ${ }^{1}$ \\ Institute of Molecular and Cell Biology, National University of Singapore, Singapore 119260
}

The $\mathbf{3 0 0}$ distinct neurons comprising each hemineuromere of the Drosophila embryonic central nervous system are derived from a segmentally reiterated array of $\sim 30$ progenitor cells, neuroblasts (NBs). Each NB has a unique identity and undergoes repeated cell divisions to produce several smaller secondary precursor cells, ganglion mother cells (GMCs); each GMC divides once to produce two neurons and/or glia, thereby generating a specific lineage of neurons/glia. Understanding the generation of neuronal diversity requires not only elucidation of the molecules and mechanisms that specify NB identity but also those that act to differentiate between the cell types produced within one NB lineage. Here we show that the Drosophila Zn finger protein Klumpfuss (Klu), which shows sequence similarities to the mammalian Wilm's tumor suppressor (WT-1), acts to differentiate between the identities of the first two secondary precursor cells produced from one NB lineage. Klu is expressed in the NB4-2 lineage only after two rounds of NB cell division, in the second born GMC (GMC4-2b). In loss-of-function mutant embryos, the first born GMC (GMC4-2a) as well as its progeny neurons are duplicated; we show that this duplication of the GMC4-2a sublineage arises because GMC4-2b adopts the identity of GMC4-2a and divides to produce the GMC4-2a progeny. Moreover, when Klu is ectopically expressed in GMC4-2a, it fails to acquire its normal identity and fails to produce correctly specified progeny. $k l u$ therefore acts to specify the identity of GMC4-2b and to make it distinct from GMC4-2a. Our findings further suggest that the determination of GMC cell fate occurs in two steps; the initial GMC identity is the consequence of inheritance from the maternal NB, however, the subsequent stabilization of this identity requires functions like klu in the GMC.

[Key Words: Drosophila embryonic CNS; neuronal diversity; neuroblast lineage; cell fate specification]

Received March 6, 1997; revised version accepted April 14, 1997.

The Drosophila embryonic central nervous system (CNS) provides an excellent model for elucidating the developmental mechanisms that lead to the genereration of neural cell diversity. The neurons and glia of the Drosophila embryonic CNS are arranged in a stereotypic pattern that is bilaterally symmetric and segmentally reiterated. The -350 distinct neurons and glial cells present in each of the hemineuromeres /cell number estimated from Ito et al. 1955; Bossing et al. 1996b) are derived from $\sim 30$ progenitor cells called neuroblasts (NBs) and glioblasts (GBs) (Campos-Ortega, 1993; Doe and Technau 1993; Goodman and Doe 1993; Jimenez and Modolell 1993; Doe and Skeath 1996). Because of the availability of a large number of enhancer trap and antibody markers

${ }^{1}$ Corresponding author.

E-MAIL MCBWCHIA@LEONIS.NUS.SG; FAX 65-779-1117.
(Doe 1992; Broadus et al. 1995), as well as their distinct morpholgy and position, many of the neurons and glial cells and essentially all of the progenitor NBs can be identified unambiguously.

NBs are formed in several temporal waves with each NB possessing a unique cellular identity that is defined by the time of its birth and its position within the neuroectoderm (Hartenstein and Campos-Ortega 1984; Doe 1992; Chu-LaGraff and Doe 1993; Skeath et al. 1995). The unique identity of each $\mathrm{NB}$ is manifested in the specific combination of genes (NB identity genes) it expresses and by the unique lineage of neurons and/or glia it generates. Each NB undergoes several asymmetric cell divisions to generate, in a distinct temporal order, a specific set of unique intermediate precursor cells called ganglion mother cells (GMCs); each GMC divides to produce two postmitotic neuron/glia. These observations suggest (see Doe 1992) that NB identity is dynamic and 
evolves in a unidirectional manner. Each GMC has a unique identity that is defined initially by determinants inherited from the parental NB and ultimately by a specific combination of expressed genes (GMC identity genes).

It has been shown that individual neuroectodermal cells can be labeled to reveal complete embryonic NB lineages (Bossing and Technau 1994). The lineages of three NBs--NB 1-1 (Udolph et al. 1993; Prokop and Technau 1994), NB 2-2 (Bossing et al. 1996a), and NB 4-2 (Chu-LaGraff et al. 1995; Udolph et al. 1995) have been delineated previously. NBl-1 was found to generate the $\mathrm{aCC} / \mathrm{pCC}$ neurons, several unidentified neurons and the A- and B-glia. These findings indicate that neurons and glia are not necessarily produced by distinct progenitors but that the decision to adopt a neural or glial fate can occur within one lineage. This type of progenitor cell can be described as neuro-glioblasts and additional progenitor cells, for example NB2-2, have similar properties. More recent work has resulted in the elucidation of the terminal lineages of $17 \mathrm{NBs}$ (Bossing et al. 1996b).

To understand the developmental mechanisms that lead to the generation of cell diversity within the CNS, it is important to define not only the genes that act to specify the unique cellular identities of each of the NBs but also genes that act to differentiate between the cellular identities that are produced within one stem cell lineage. For this purpose, we have focused on NB4-2, genetically the best characterized NB lineage in the Drosophila embryonic CNS (see Fig. 1). The first-born GMC of NB4-2, designated GMC4-2a (nomenclature according to Doe 1992; see also legend to Fig. 1), divides to produce the RP2 motoneuron and a sibling cell. A number of useful molecular markers for GMC4-2a and the RP2 neuron have been described (Fig. 1).

Several genes have been described previously whose activities are required for the generation of a correctly specified RP2 neuron. The secreted gene product of wingless (wg) (Chu-LaGraff and Doe 1993) is required for the formation and specification of NB4-2. In addition, the homeobox genes fushi tarazu (ftz) and even-skipped (eve) (Doe et al. 1988a,b) and the POU domain genes pdm-1 and pdm-2 (Billin et al. 1991; Dick et al. 1991; Lloyd and Sakonju 1991) are expressed in GMC4-2a and are necessary for specifying its cellular identity and for the formation of the RP2 neuron (Bhat et al. 1995; Yeo et al. 1995). The homeobox gene prospero (pros) (Doe et al. 1991; Matsuzaki et al. 1991; Vaessin et al. 1991) is expressed on the basal cortex of many dividing NBs and inherited by their GMC progeny (Hirata et al. 1995; Knoblich et al. 1995; Spana and Doe 1995), including GMC42a. Total loss of pros function affects the identities of many GMCs and neurons including those of GMC4-2a and RP2. In addition, the putative transcription factor Huckebein appears to regulate aspects of GMC and neuronal identity required for the correct differentiation of motoneurons arising from the NB4-2 lineage (Chu-LaGraff et al. 1995) as well as for glial development and axon pathfinding in the NB1-1 and NB 2-2 lineages (Bossing et al. 1996a).
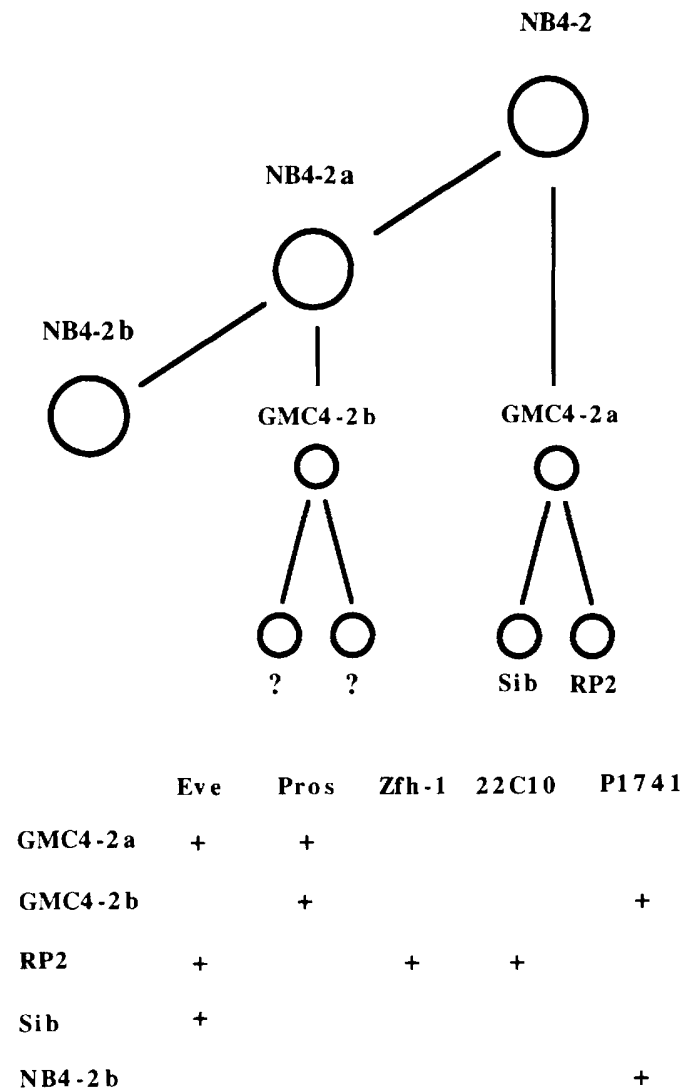

Figure 1. The early born cells of the NB4-2 lineage. This diagram depicts the cells derived from the first two NB divisions and the antibody and enhancer trap markers used in this study for their identification. NB4-2a and GMC4-2a are the progeny of NB4-2 after its first asymmetric division. GMC4-2a divides to produce the RP2 motoneuron and its sibling cell. NB4-2b and GMC4-2b are the progeny of the NB4-2a cell division, the second NB division in this lineage. The identities of the progeny of GMC4-2b are not known. Anti-Eve labels cells in the GMC4-2a sublineage. Anti-Pros marks all GMCs including GMC4-2a and GMC4-2b. klu ${ }^{p 174 l}$ expresses $\beta-G a l$ in NB4-2b and GMC4-2b. RP2 neuron is also labeled by both anti-Zfhl and mAb $22 \mathrm{C} 10$.

In a screen designed to identify mutations that alter RP2 cell number, we isolated loss-of-function mutations of klumpfuss, (klu), which encodes a Wilm's tumor suppressor (WT-1)-like Zn finger protein (Klein and CamposOrtega 1997). Here we demonstrate that Klu protein expression is nuclear and can be seen in many NB lineages; within the NB4-2 lineage, Klu protein expression commences only following two rounds of NB cell division in the GMC4-2b nucleus. In klu loss-of-function mutant embryos, the RP2 motoneuron appears to be duplicated; clonal analysis indicates that the duplicated neurons arise within the NB4-2 lineage. By following the fates of GMC4-2b and GMC4-2a in mutant animals, we show that loss of klu function causes a GMC4-2b to GMC4-2a cell fate transformation; thereby duplicating the GMC42 a sublineage. Furthermore, we demonstrate that misexpression of Klu in GMC4-2a, which normally does not 
express Klu, prevents it from adopting a correct GMC4$2 \mathrm{a}$ cell identity and prevents the formation of correctly specified RP2 neurons. Taken together, our work demonstrates that klu acts to differentiate between the identities of the first two GMCs generated within the NB4-2 lineage; its expression in the second-born GMC, GMC4$2 b$, is necessary to specify the identity of this cell, making it distinct from the first-born GMC, GMC4-2a.

\section{Results}

\section{The klu mutant alleles}

We isolated the enhancer trap line P1741 from an anti$\beta$-Gal screen of lethal enhancer trap lines provided by the Bloomington Stock Center. From 500 enhancer trap lines screened, 37 lines with $\beta$-Gal expression in all or subsets of NBs were identified. P1741 /cytological position 68A1-2) showed an RP2 duplication phenotype (see below). P1741 (klu $\left.{ }^{P 1741}\right)$ was inserted in klu that had been cloned and for which a number of mutant alleles already existed (Klein and Campos-Ortega 1997). A summary description of the nature of the klu alleles used in this study and their molecular defects are given (Fig. 2). The various alleles show similar CNS defects (see below) at different expressivity. Our phenotypic analysis focused primarily on two null alleles of $k l u ; D f(3 R) k I u^{X R I 9}$ is a one band (cytological) deficiency obtained as a $w^{-}$ revertant induced by X-rays; $k l u^{p 212 I R 51 C}$, generated by the imprecise excision of $k l u^{P 212}$, a $\mathrm{P}$ element inserted near the 3 '-end of the klu transcription unit, contains a small, molecularly mapped, deletion that removes at least three exons from the 3 '-end of klu IT. Klein and J.A. Campos-Ortega, in prep.). Both of these alleles are antigen-minus when stained with an antibody raised against a Klu fusion protein (see Materials and Methods).

Klu protein is expressed in the developing embryonic CNS

The temporal and spatial expression pattern of Klu has been characterized with an anti-Klu antibody (Fig. 3) and is essentially identical to the anti- $\beta$-Gal pattern observed in both the klu $u^{P 174}$ and $k l u^{P 212}$ enhancer trap insertions (data not shown). Consistent with its putative role as a transcription regulator, the antibody shows nuclear staining in embryos. The earliest Klu expression is nuclear and occurs before syncitial blastoderm (Fig. 3A). The staining disappears after cellularization. At stage 10, anti-Klu staining reappears in the nuclei of a subset of $\mathrm{NB}$ and in the procephalic region. The staining becomes more prominant in the NBs and brain at stage 11 ; some epithelial cells start to express Klu and are stained as patches on the lateral sides of the embryos (Fig. 3B). The staining in the ventral cord and brain continues at stage 12. At stage 16, the staining seen in the ventral cord and the brain remains but is weaker; the epithelial cells are stained as three- to four-cell-wide stripes; the posterior spiracles are also $\mathrm{Klu}^{+}$(Fig. 3C,D).

The expression of Klu in neuroblasts is shown (Fig. 3E-I). The earliest NB staining (nuclear) is seen at stage 10 (SIII), when six NBs are initially stained by the antibody (Fig. 3E,H); they are row 5 NBs (NB5-2, NB5-3, and NB5-6); row 7 NBs (NB7-1 and NB7-4), and NB3-5. At stage 11 (SIV), with the exception of the five NBs (NB2-4, NB3-3, NB4-4, NB5-4, and NB6-4), all NBs show nuclear staining (Fig. 3F,I). At late stage 11 (SV), only two NBs (NB2-3 and NB6-4) do not express Klu (Fig. 3G,J). Klu is also expressed in some GMCs (see below).

\section{RP2 neuron is duplicated in klu embryos}

To detect possible cell fate changes and defects in the developing embryonic CNS of klu mutant embryos, an array of marker antibodies were used, including anti-Eve (Frasch et al. 1986), anti-Ftz (W. Gehring laboratory), anti-En (Patel et al. 1989b), anti-Zfh1 (Lai et al. 1991), mAbBP102 (C. Goodman laboratory), mAb 22C10 (Fujita et al. 1982), and mAblD4 (Van Vactor et al. 1993). The only marker antibodies that gave obviously altered stain-
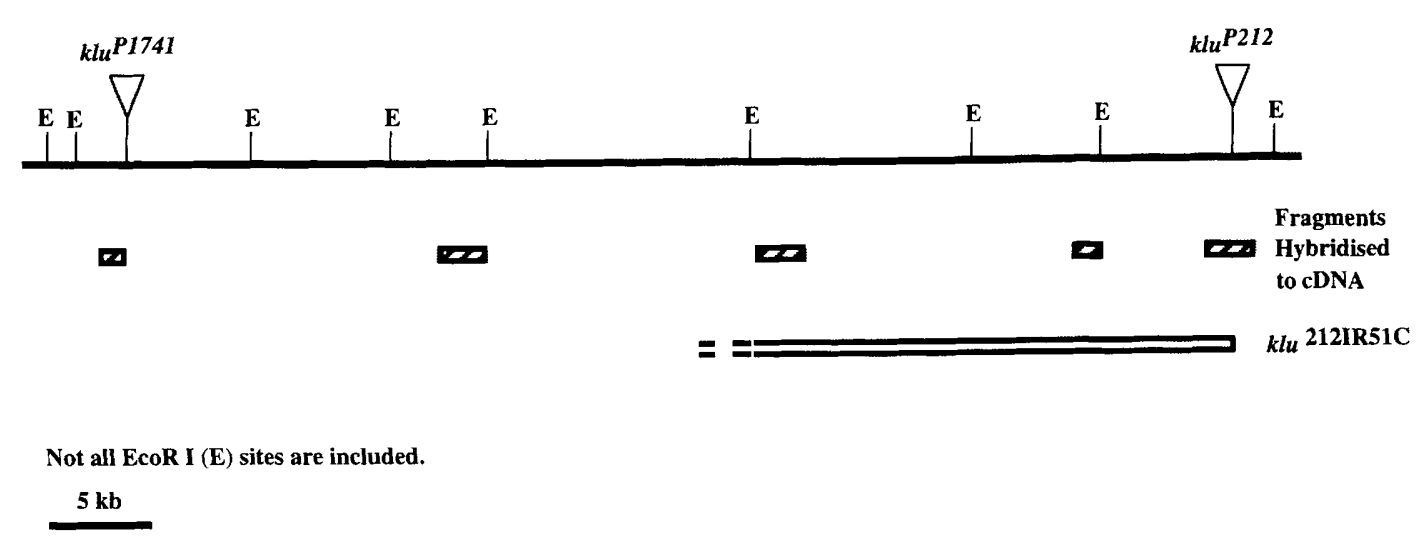

Figure 2. The molecular lesions associated with two klu alleles. Genomic DNA spanning $63 \mathrm{~kb}$ that contains the transcribed region of $k l u$ is shown. The regions that hybridize to a full-length klu cDNA are indicated on the map. The klu transcribed region contains at least five exons. The positions of the insertion sites of $k^{\prime} u^{P 1741}$ and $k I u^{P 212}$ are indicated. klu ${ }^{212 I R 51 C}$ removes all or part of the three $3^{\prime}$-most exons; its 5 ' breakpoint lies within a 3 -kb region (double dash line); $D f(3 R) k l u^{X R 19}$ (data not shown) deletes one cytological band and removes the entire region shown here (Klein and Campos-Ortega 1997) 

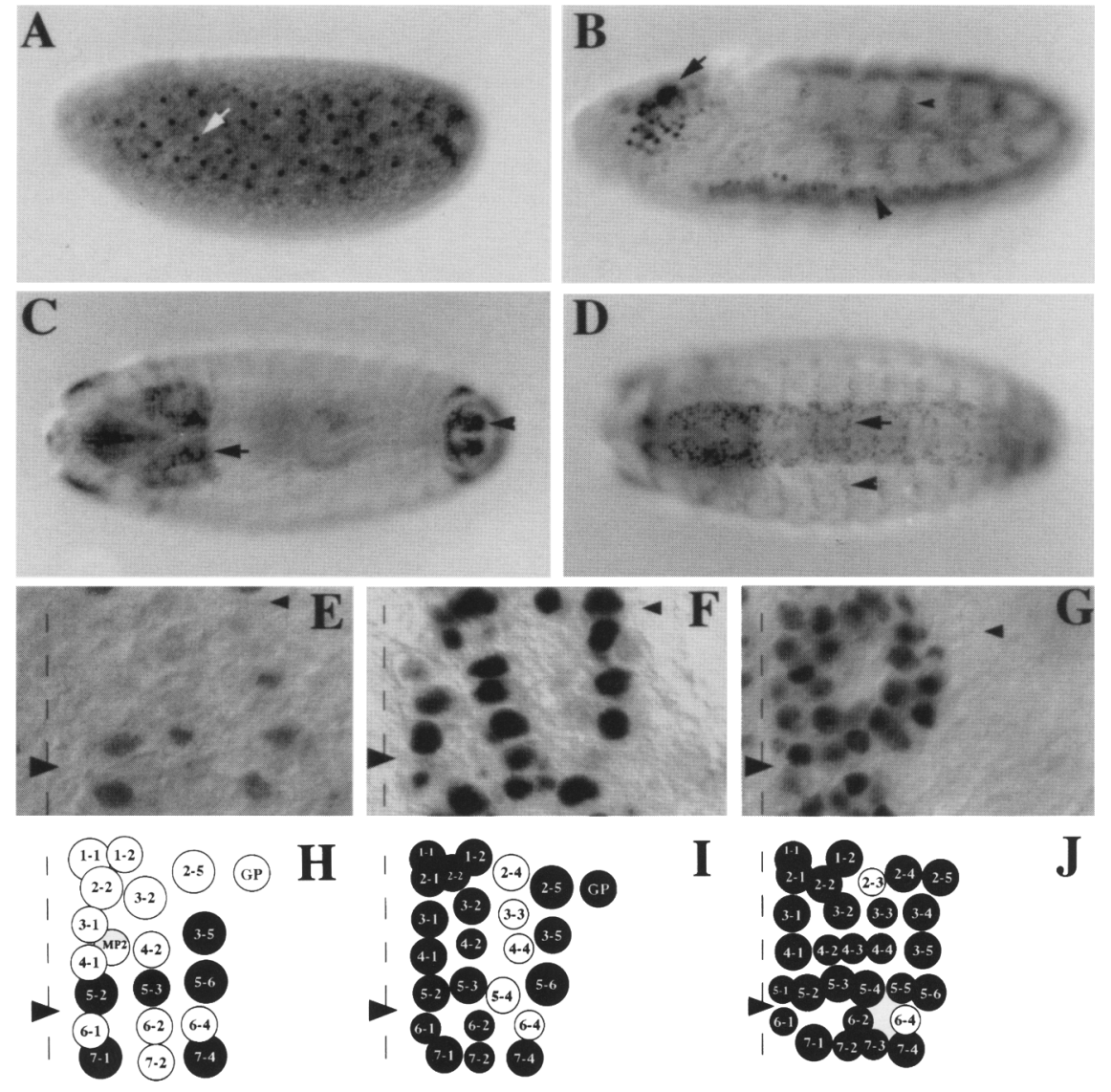

Figure 3. The expression pattern of Klu protein in the embryo and in identified neuroblasts during early CNS development. $(A)$ Lateral view of a pre-blastoderm embryo showing Klu protein expression in syncitial nuclei (arrow) before cellularization. (B) Lateral view of a stage-1 1 embryo showing Klu protein expression in the nuclei of the procephalic region (arrow), patches of epithelial cells (small arrowhead) and cells of the developing CNS (arrowhead). (C) Dorsal view of a stage-16 embryo. Nuclei of cells in the brain lobes (arrow) and posterior spiracles (arrowhead) are stained by anti-Klu. (D) Ventral view of same embryo seen in $C$. Nuclear staining in cells of the ventral cord (arrow) and stripes of epithelial cells (arrowhead). (E$G)$ Klu expression pattern in identified neuroblasts during three stages of NB segregation. Ventral views of one hemisegment each of a stage-10 (following SIII segregation, $E$ ), a stage-11 (following SIV segregation, $F$ ) and a late stage- 11 (following SV segregation, $G$ ) embryo are shown, focused at the level of the NBs. The Klu expression patterns shown in $E-G$ are illustrated diagrammatically in $H-I$, respectively; Klu-expressing NBs are in black. For $A-D$, anterior is left; dorsal is up in $A$ and $B$. For $E-J$, anterior is up; dashed line indicates the midline and arrowhead marks the parasegmental groove. ing patterns in klu mutant embryos were anti-Eve, mAb $22 \mathrm{C} 10$ and anti-Zfhl, markers that are expressed in the RP2 neuron (see Fig. 1). The anti-Eve antibody stains $\sim 20$ neurons in each embryonic hemineuromere (Patel et al. 1989a) and is a particularly useful marker for the early part of the NB4-2 lineage (Fig. 1). During neurogenesis, NB4-2 divides to produce its first ganglion mother cell (GMC4-2a) at stage 10. The GMC4-2a becomes Eve ${ }^{+}$and undergoes terminal division to produce two Eve ${ }^{+}$cells at stage 11. One of the progeny cells retains Eve expression (until the end of embryogenesis) and differentiates into the mature RP2 motoneuron with its characteristic position in the CNS scaffold, anterior lateral axon projection, and expression of marker genes by stage 13. RP2 sibling cell loses Eve expression completely at stage 13 and its final fate is unknown.

When stage-15 klu embryos were stained with antiEve, we observed two Eve ${ }^{+}$cells per hemisegment (Fig. $4 \mathrm{~B})$, compared with only one $\mathrm{Eve}^{+}$cell per wild-type hemisegment (Fig. 4A), at the characteristic RP2 position in the embryonic CNS. The RP2 duplication phenotype is position dependent; the frequency of duplication in $\mathrm{T} 2, \mathrm{~T} 3$, and A1 segments is much higher than that in the other abdominal segments (A2-A8). The anteriormost neuromeres are difficult to score at late stages because of curvature of the CNS. The percentage of hemisegments containing two Eve $^{+}$cells at RP2 position varied among the different mutant alleles. For the $k l u^{P 1741}$ homozygotes, $49 \%(118 / 240)$ of the T2-Al hemisegments showed duplicated RP2 neurons but no duplications $10 /$ $560)$ were seen in the A2-A8 hemisegments. In $\mathrm{K}^{1} \mathrm{u}^{\text {p2121R51C }}$ homozygotes, $99 \%,(238 / 240)$ of the T2-A1 hemisegments exhibited duplicated RP2 neurons; the RP2 duplication also occurred in A2-A8 (26\%; 145/560), but at a lower frequency. Homozygotes for $D f(3 R) k l u X R 19$, which removes the entire klu transcription unit, showed similar expressivity [T2-A1: $99 \%(238 / 240)$; A2-A8: $22 \%(123 / 560)]$ to $k l u^{p 212 I R 51 C}$, indicating that $k l u^{p 212 I R 51 C}$ is an amorphic allele. The majority of our analyses are based on $\mathrm{klu}^{\mathrm{p} 2121 \mathrm{R} 51 \mathrm{C}}$ unless otherwise specified.

Both of the Eve ${ }^{+}$cells at the RP2 position in klu embryos express additional markers consistent with a RP2 identity. Zfh 1 is a $\mathrm{Zn}$ finger homeodomain protein, expressed in a variety of tissues in the embryos, including CNS neurons and muscles (Lai et al. 1991). Zfhl protein is expressed in the RP2 and aCC motoneurons starting stage 13. mAb 22C10 (Fujita et al. 1982) labels the membrane of a subset of CNS neurons including the RP2 motoneuron. Like their wild-type counterpart (Fig. $4 A, C, E$ ), the duplicated RP2 neurons are stained by $\mathrm{mAb}$ 22C10 (Fig. 4D) and anti-Zfhl (Fig. 4F); their axon projections appear to be in an anterior ipsilateral direction (Fig. 4D), identical to those of wild-type RP2 neurons (Fig. 4C). Therefore, we conclude that both $\mathrm{Eve}^{+}$cells at 
Figure 4. In the klu mutant embryonic CNS, two RP2-like neurons are produced from the NB4-2 lineage. $\{A, B\}$ Dorsal views of dissected wild-type $(A)$ and klu mutant $(B)$ stage-15 embryos stained with anti-Eve antibody; whereas only a single Eve ${ }^{+}$RP2 neuron (arrow) is seen per hemisegment in wild type, two eve ${ }^{+}$cells (arrows in $B$ ) per hemisegment are present at the characteristic RP2 position in the mutant embryonic CNS. $\{C, D\rangle$ Dorsal views of dissected wildtype $(C)$ and mutant $(D)$ stage-15 embryos double-labeled with anti-Eve (black, nuclear staining) and $\mathrm{mAb} 22 \mathrm{Cl} 0$ (brown, cell surface and axon staining). The wildtype RP2 neuron (arrow, $C$ ) is labeled by both antibodies and exhibits an ipsilateral axon projection (arrowhead). Note that in the mutant embryo, both of the Eve ${ }^{+}$cells at the RP2 position (arrows, D) are stained by anti-Eve and $\mathrm{mAb} 22 \mathrm{ClO}$ and the ipsilateral axon projection (arrowhead) appears to be identical to that seen in wild type.
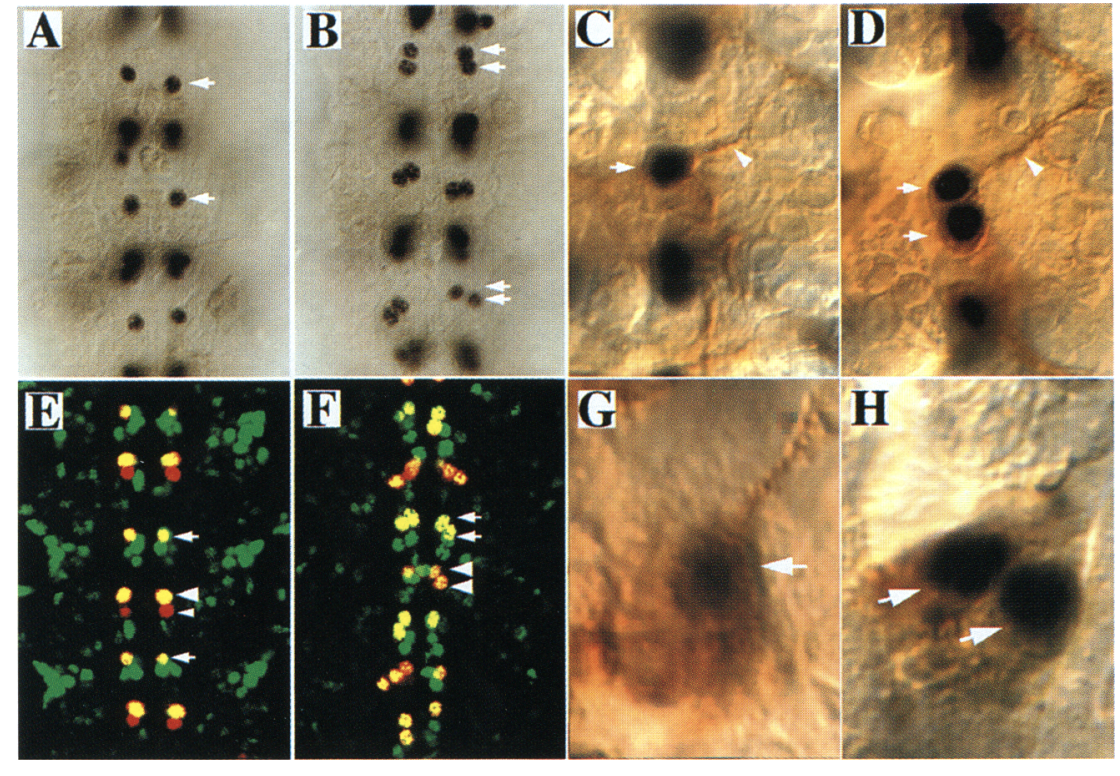
cal images of wild-type $(E)$ and mutant $(F)$ stage-15 embryos stained with anti-Eve (red) and anti-Zfhl (green). In wild-type hemisegments $(E)$, the RP2 neuron (arrow) is double positive (yellow) for Eve and Zfh 1; the anterior corner cell (aCC, arrowhead) is also double positive (yellow) whereas the posterior corner cell (pCC, small arrowheadl is positive only for Eve (red). In mutant hemisegments $\{F$ ), both of the Eve ${ }^{+}$cells at the RP2 position (arrows), like the wild-type RP2 neuron, also express Zfhl (yellow). Note that whereas the wild-type pCC (small arrowhead in $E$ ) expresses Eve but not Zfhl (red), the mutant pCC (lower arrowhead in $F$ ) often expresses both Eve and Zfhl (yellow). $\{G, H)$ Dorsal views of Tau- $\beta$-Gal expressing clones (see text and Materials and Methods) from dissected wild-type $(G)$ and mutant $(H)$ embryos double stained with anti- $\beta$-Gal (membrane and axon staining, brown) and anti-Eve (nuclear staining, black), focused at the level of the RP2 neuron. A single Tau- $\beta$-Gal and Eve double positive RP2 $\{G$, arrow $\}$ is seen from a wild-type NB4-2 clone. A clone generated from a mutant embryo includes both RP2 neurons (arrows in $H$ ), indicating that the duplicated RP2 neurons are derived from the same NB4-2 lineage. Anterior is up.

the RP2 position show characteristics of mature RP2 neurons.

\section{The duplicated RP2 neurons are derived from the same NB lineage}

As a first step in understanding the origin of the duplicated RP2 neurons, we examined their lineage relationship. Clonal analysis in klu embryos demonstrates that both RP2 neurons are derived from the same lineage. For these experiments, the actin-tau-lacZ gene in klu mutant embryos containing both the actin-tau-lac $Z$ "flip cassette" and HS-flippase constructs can be permanently activated by controlled heat-shock treatment (see Materials and Methods). If the activation of actin-taulac $Z$ occurs in an NB before its first division, all progeny cells derived from that NB will express tau-lacZ and form a $\beta-\mathrm{Gal}^{+}$clone in the ventral cord. Clones containing RP2 neurons can be easily identified by staining the embryos with anti-Eve and anti- $\beta$-Gal. We found three such clones with duplicated RP2 neurons in stage 15-16 klu embryos and, in all cases, both RP2 neurons were labeled by anti- $\beta$-Gal antibody together with other $\beta-\mathrm{Gal}^{+}$progeny cells (Fig. $4 \mathrm{H}$ ). In two of these cases, the clones appeared to be about the same size as wild-type NB4-2 clones. These results (in combination with other results shown below) clearly indicate that both RP2 cells in klu embryos are derived from the NB4-2 lineage.

klu expression within the NB4-2 lineage commences following two rounds of NB cell division, in GMC4-2b and NB4-2b

To elucidate the cell fate transformation that must have occurred within the NB4-2 lineage to generate the duplicated RP2 neurons, we first assessed the expression of Klu within this lineage. Pros is expressed essentially in all nuclei of GMCs in the developing CNS and can be used as a general GMC marker. At late stage 10 (SIII), anti-Pros antibody staining shows that there is a Pros ${ }^{+}$ cell dorsal to NB4-2 (Fig. 5C), indicating that the first round of NB4-2 cell division is complete and GMC4-2a is formed at this stage; the neuroblast seen at the NB4-2 position at this time is NB4-2a; anti-Klu and anti-Pros double-labelings indicate that NB4-2a and GMC4-2a are both $\mathrm{Klu}^{-}$(Fig. 5A-F). Double labeling of stage-11 (SIV) embryos with anti-eve and anti-Klu antibodies confirms that GMC4-2a, which has by this stage commenced Eveexpression, is not labeled by anti-Klu (Fig. 5E); interestingly, there is a small $\mathrm{Klu}^{+}$cell just dorsal to the $\mathrm{Klu}^{+}$ NB4-2b and close to GMC4-2a (Fig. 5F). Further experiments show that this $\mathrm{Klu}^{+}$cell is also labeled by the 

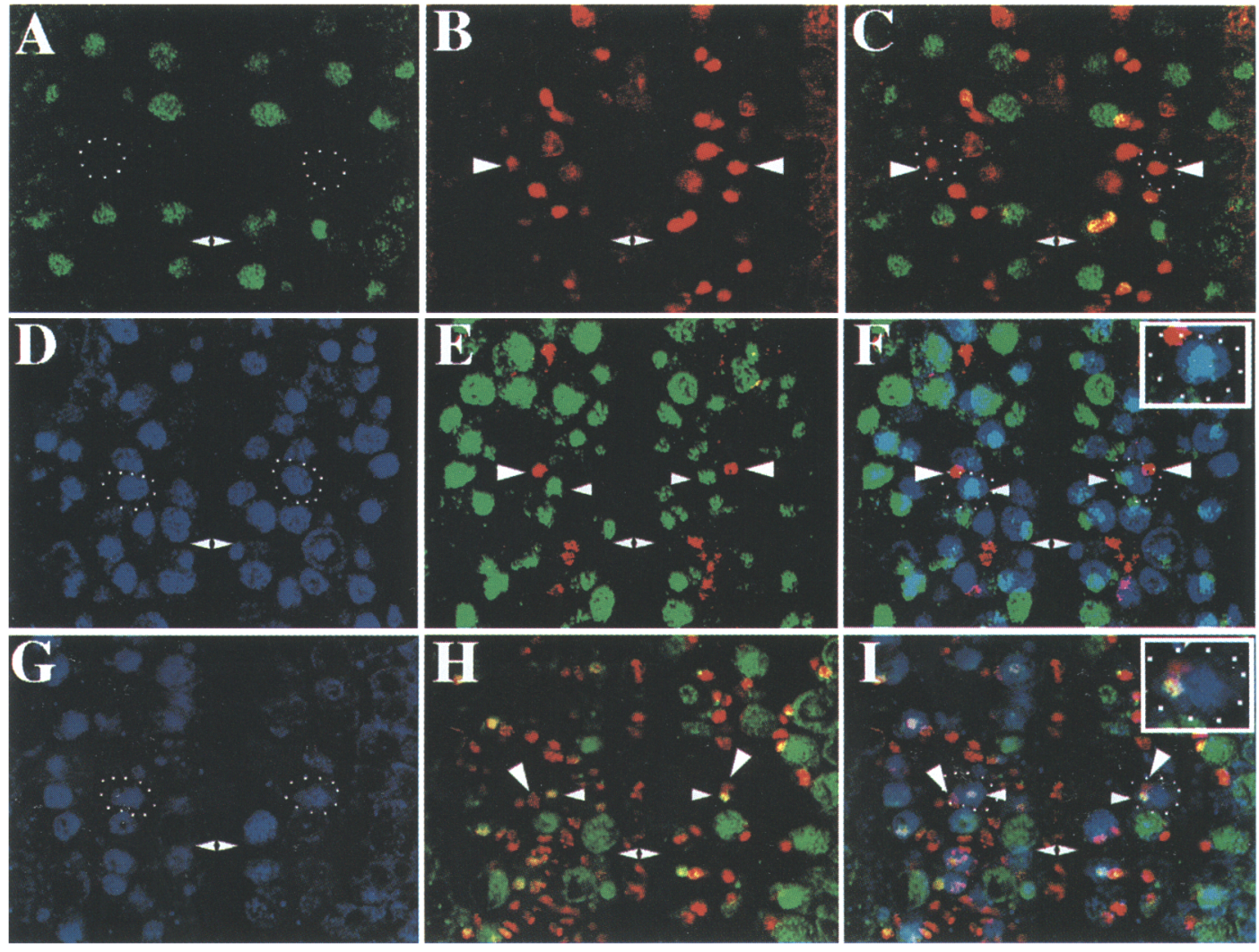

Figure 5. Klu protein expression within the NB4-2 lineage. Confocal images of an anti-Klu and anti-Pros double-labeled stage-10 embryo $(A-C)$, an anti-Klu and anti-Eve double-labeled stage- 11 embryo $|D-F|$, and an anti-Klu and anti-Pros double-labeled stage-11 embryo $(G-I)$. $(A)$ Klu expression in NBs (green). Note that NB4-2a (circled by dots) does not express Klu at this stage. $(B)$ Pros expression (red) in the same embryo shown in $A$ but focused at the GMC level; GMC4-2a (arrowhead) is formed. $(C)$ Superimposed image of $A$ and $B$; GMC4-2a is located just dorsal to NB4-2a (circled by dots). (D) Klu expression (blue) at stage 11 (SIV segragation), focusing at the NB level. NB4-2b (circled by dots) is Klu+. (E) Superimposed image of anti-Klu (green) and anti-Eve (red) double-staining of the same embryo shown in $D$ but focused at the GMC level; GMC4-2a is Eve (large arrowhead, red) and GMC4-2b is $\mathrm{Klu}^{+}$(small arrowhead, green). $(F)$ Overlay of $D$ and $E$, showing that at SIV segregation, when only two GMCs are dorsal to NB4-2b (circled by dots), one of them is GMC4-2a (red, Eve ${ }^{+}$) and the other is GMC4-2b (light blue, $\mathrm{Klu}^{+}$; an enlargement of the relevant region is shown in the inset. (G) Klu expression (blue) at SIV, focusing at the NB level; NB4-2b (circled by dots) is Klu ${ }^{+}$. $(H)$ Superimposed images of the same embryo shown in $G$ double-labeled for Klu (green) and Pros (red), focused at the GMC level. (I) Overlay of $G$ and $H$, showing that at SIV only two GMCs are dorsal to NB4-2b: GMC4-2a (Pros ${ }^{+} \mathrm{Klu}$, red, large arrowhead) and GMC4-2b (Pros ${ }^{+} \mathrm{Klu}^{+}$, yellow, small arrowhead); an enlargement of the relevant region is shown in the inset. Anterior is up. The inverted double arrowheads mark the positions of the parasegmental grooves and the midline.

anti-Pros antibody (Fig. $5 \mathrm{H}$ ), revealing its identity as a GMC. Because there are only two Pros ${ }^{+}$GMCs dorsal and in close proximity to NB4-2b (at SIV), and one of

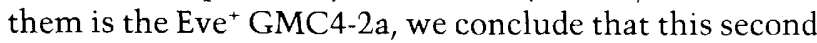
$\mathrm{Klu}^{+}$and Eve ${ }^{-}$cell is GMC4-2b. Therefore, these data indicate that the earliest expression of Klu within the NB4-2 lineage occurs in GMC4-2b and NB4-2b, after the NB has undergone two rounds of cell division.

\section{Transformation of GMC4-2b to GMC4-2a in klu embryos}

Both the klu mutant phenotype and the Klu expression pattern are consistent with the possibility that the extra RP2 neuron found in the mutant results from a GMC4$2 \mathrm{~b}$ to GMC4-2a cell fate transformation; to ascertain whether the mutant GMC4b does in fact behave like GMC4-2a, that is, express markers consistent with a GMC4-2a identity and divides like GMC4-2a to produce another RP2 and another sibling cell, we require a marker to follow GMC4-2b in klu mutants. The absence of an antigen-positive mutant allele of $k l u$ precludes the use of anti-Klu as a marker for the mutant GMC4-2b. Nevertheless, we reasoned that it should be possible to use the $k l u^{I^{\prime} 1741}$ enhancer trap allele to follow the fate of the mutant GMC4-2b. The klu ${ }^{P 1741} \beta$-Gal expression pattern is identical to that of anti-Klu staining (data not shown) and $\beta$-Gal expression can be detected in GMC4$2 \mathrm{~b}$ and not in GMC4-2a (see Fig. 5). Moreover, klu $\mathrm{u}^{P 1741}$ homozygotes exhibit the RP2 duplication phenotype at a reasonably high expressivity $(49 \%$ in $\mathrm{T} 2-\mathrm{A} 1)$. We reasoned that because the initial identity of GMC4-2b 
should be determined by the identity of its mother, NB4$2 \mathrm{a}$, the mutant GMC4-2b in klu mutants should be born with an GMC4-2b identity because NB4-2a does not express Klu and its identity should be unaffected in the mutant; GMC4-2b should therefore initially express $\beta$-Gal even in a mutant background (e.g., klup1741) $k l u^{P 1741}$; the $\beta$-Gal that is produced should perdure and continue to mark the mutant GMC4-2b (and its progeny) even if it were to adopt a GMC4-2a identity later attributable to limiting klu function. Therefore, we should be able to follow the fate of the mutant GMC4-2b in the $k 1 u^{P 1741}$ homozygote by virtue of the $\beta$-Gal expression and perdurance.

Results of double labeling $k l u^{p 1741}$ embryos with anti-
$\beta$-Gal and anti-Eve are shown in Figure 6. In wild-type control embryos, [klup1741/TM3(ubx-lacZ)], GMC4-2a becomes $\mathrm{Eve}^{+}$at stage 11 (SIV) (Fig. 6A) and later divides to produce two $\mathrm{Eve}^{+}$postmitotic neurons (Fig. 6B). One of the postmitotic neurons continues to express Eve and differentiates into the RP2 neuron and the other (the sibling cell) extinguishes Eve expression (Fig. 6C,D). In control embryos, no more than two $\mathrm{Eve}^{+}$cells are seen in the RP2-sib cluster and neither are labeled by anti- $\beta$-Gal.

In klu ${ }^{P 1741}$ homozygous embryos, GMC4-2a becomes $\mathrm{Eve}^{+}$at stage 11 (Fig. 6F), same as in wild-type (Fig. 6A); later, a second $\mathrm{Eve}^{+}$cell that is also labeled by the anti$\beta$-Gal antibody appears in the close proximity to GMC4 $2 a$ dorsal to NB4-2b (Fig. 6G). Anti- $\beta$-Gal labeling of one
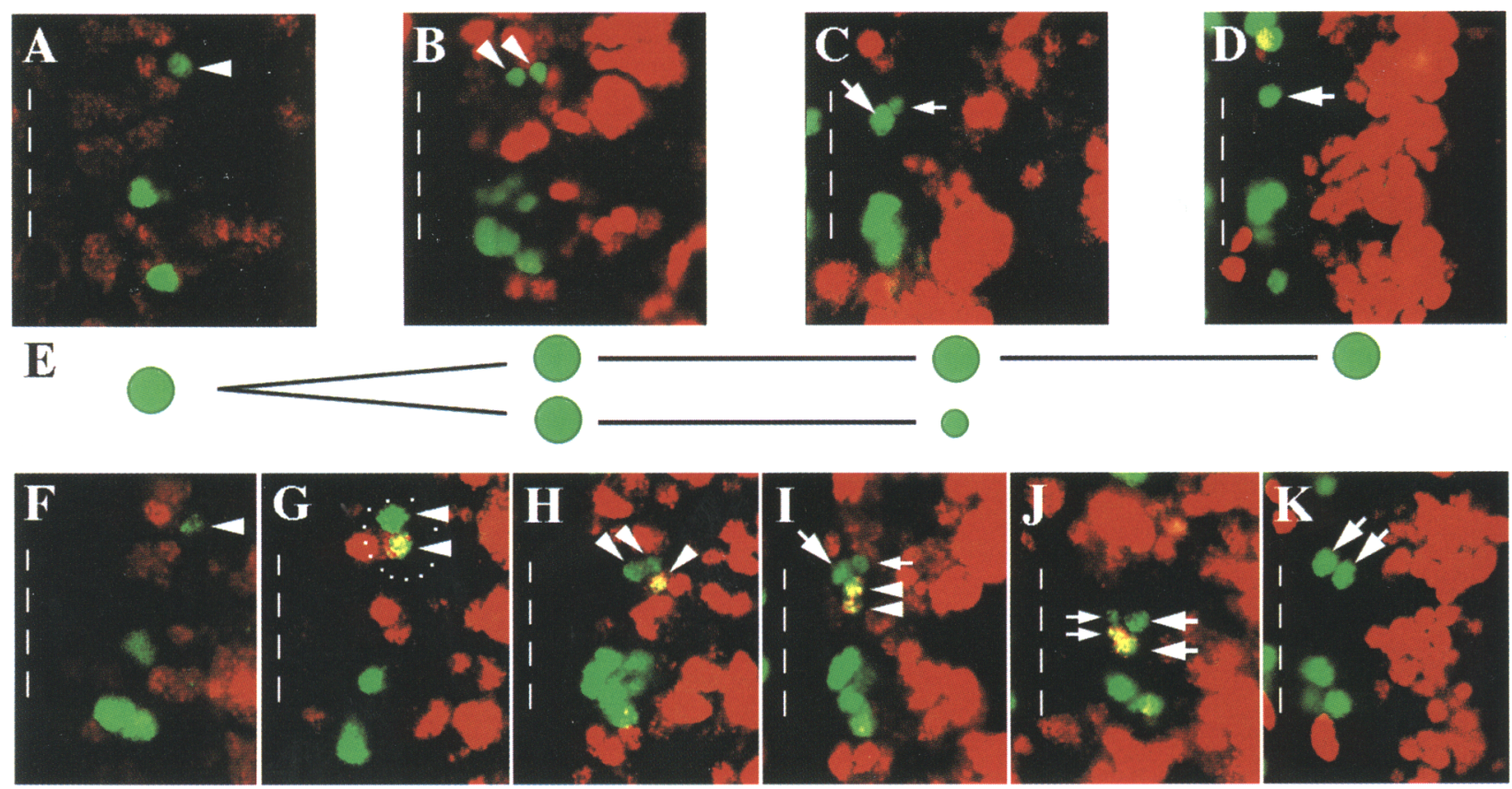

$\mathbf{L}$
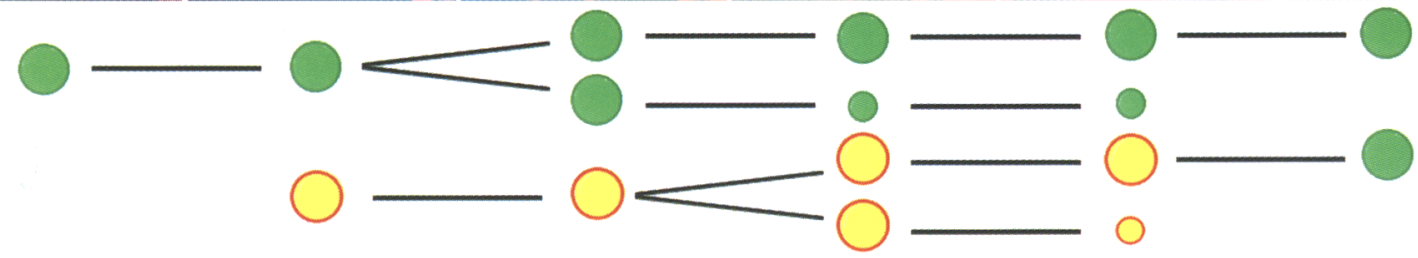

Figure 6. GMC4-2b adopts GMC4-2a identity in the enhancer trap line klu ${ }^{p 1741}$. The embryos were double labeled with anti- $\beta$-Gal

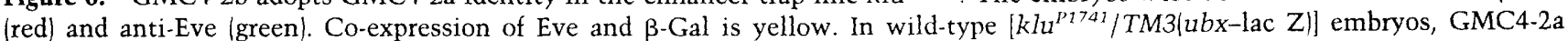
(arrowhead, $A$ ) becomes Eve ${ }^{+}$at stage 11 ; later it divides to produce two Eve ${ }^{+}$postmitotic neurons (arrowheads, $\left.B\right)$. At stage $12(C)$, one of the $\mathrm{Eve}^{+}$cells continues to express Eve and differentiates into the RP2 neuron (arrow), whereas the sibling cell (small arrow) loses Eve expression gradually. By stage 15 only the mature RP2 remains Eve ${ }^{+}$(arrow, $D$ ). A diagram summarizing the process of RP2 formation seen from A-D is shown $\{E\rangle$. In $k l u^{p 1741}$ homozygotes, GMC4-2a (arrowhead, green) becomes Eve ${ }^{+}$at stage $11\langle F\rangle$ as in wild-type $(A)$. Shortly after that, the transformed GMC4-2b (arrowhead, yellowl can be seen that is Eve ${ }^{+}$and $\beta$-Gal ${ }^{+}(G)$. Dotted circle in $G$ indicates the position of NB4-2. Later three Eve ${ }^{+}$cells are seen in the cluster $(H)$ : two $\beta$-Gal ${ }^{-}$postmitotic neurons generated by GMC4-2a (green) as in wild type and the transformed $\beta-\mathrm{Gal}^{+}$GMC4-2b (yellow). At late stage 11, there are four Eve ${ }^{+}$cells in the cluster (I): two $\beta-\mathrm{Gal}^{+}$postmitotic neurons (yellow) derived presumably from the transformed GMC4-2b and the $\beta-\mathrm{Gal}^{-} \mathrm{RP2}$ and sibling cell (green) derived from GMC4-2a. At stage 12, two RP2 (arrow) and two sibling cells (small arrow) are obvious (J). Note that one set of RP2 and sibling cell, presumably derived from the transformed GMC4-2b, are $\beta$-Gal- (yellow). At stage 15 , two mature Eve ${ }^{+} \mathrm{RP}^{-} \mathrm{s}$ are seen $(K)$; note that $\beta$-Gal is no longer present at this late stage. The probable process of formation of two RP2 neurons in klu embryo as seen from $F-K$ is diagrammatically summarized $(L)$. Anterior is up. Dashed line marks the midline. (Arrowhead) Eve ${ }^{+} \mathrm{GMCs}$ and postmitotic neurons. (Arrow) RP2 neuron. (Small arrow) Sibling cell. 
of the two $\mathrm{Eve}^{+}$cells indicates that these two cells are not the postmitotic neurons derived from the GMC4-2a as seen in Figure $6 \mathrm{~B}$. This $\mathrm{Eve}^{+}$and $\beta-\mathrm{Gal}^{+}$cell is the same cell that we identified earlier, using anti-Eve and anti-Klu double labelings, as GMC4-2b (see Fig. 5). In klu embryos, the mutant GMC4-2b ("GMC4-2b") adopts GMC4-2a identity and becomes Eve ${ }^{+}$; GMC4-2a divides first and gives rise to two $\mathrm{Eve}^{+}$postmitotic neurons (Fig. $6 \mathrm{H})$; at late stage 11 , "GMC4-2b" also divides and produces two postmitotic neurons that are both Eve and $\beta-\mathrm{Gal}^{+}$and at this time Eve expression starts to fade in the sibling cell produced from the GMC4-2a division (Fig. 6I); the progeny of the "GMC4-2b" cell division behave in an identical way to those of the GMC4-2a cell division with respect to Eve expression and by stage 12 , two RP2s and two sibling cells become obvious (Fig. 6J); the RP2 and sibling cells derived from the "GMC4-2b" are also $\beta-\mathrm{Gal}^{+}$. Ultimately, Eve expression becomes totally extinguished from both sibling cells, $\beta$-Gal becomes undetectable in the "GMC4-2b" sublineage and two indistinguishable Eve ${ }^{+} \mathrm{RP} 2$ neurons are seen at stage 15 (Fig. 6K).

These results, schematically represented in Figure 6L, indicate that in klu embryos two RP2 neurons are produced by two different GMCs-GMC4-2a and transformed "GMC4-2b". The "GMC4-2b" sublineage in klu embryos, which is labeled by anti- $\beta-$ Gal, is an exact, although delayed, replica of the GMC4-2a sublineage. We conclude from these results that klu functions to specify GMC4-2b identity and in the absence or under limiting klu function, GMC4-2b takes on a GMC4-2a identity.

\section{Ectopic expression of Klu reduces the number of Eve $e^{+}$ $R P 2$ neurons}

Two observations- (1) Klu is expressed in GMC4-2b but not GMC4-2a and (2) in klu mutants, GMC4-2b takes on a GMC4-2a identity-argue that klu is not only required to specify GMC4-2b identity but also acts to differentiate between the cellular identities of GMC4-2a and GMC4-2b. Therefore, we might expect that if Klu were to be ectopically expressed in GMC4-2a, where it is not normally expressed, GMC4-2a might undergo the opposite cell fate transformation leading to its loss, thereby eliminating RP2 and its sibling from the NB4-2 lineage. We set out to test these predictions.

First we looked to see if the ectopic expression of Klu causes the loss of Eve-expressing RP2 neurons. In embryos homozygous for a HSP70-klu transgene (see Materials and Methods), ectopic Klu protein induced by a 10 -min heat shock at $37^{\circ} \mathrm{C}$ became obvious within 30 min and reached a maximum level within an hour, at $25^{\circ} \mathrm{C}$; the Klu protein remained in the heat-shocked embryos for another hour and was gradually degraded afterward (data not shown). The extent to which Eve ${ }^{+}$RP2 neurons are lost depends on the developmental stage at which the heat-shock is administered (Fig. 8, see below). Embryos heat-shocked at early stage $10(4.5 \mathrm{hr}$ after egg laying) showed the maximum inhibition of $\mathrm{Eve}^{+} \mathrm{RP} 2$ formation (Figs. $7 \mathrm{~B}$ and 8$),-83 \%(99 / 120)$ of the hemiseg-
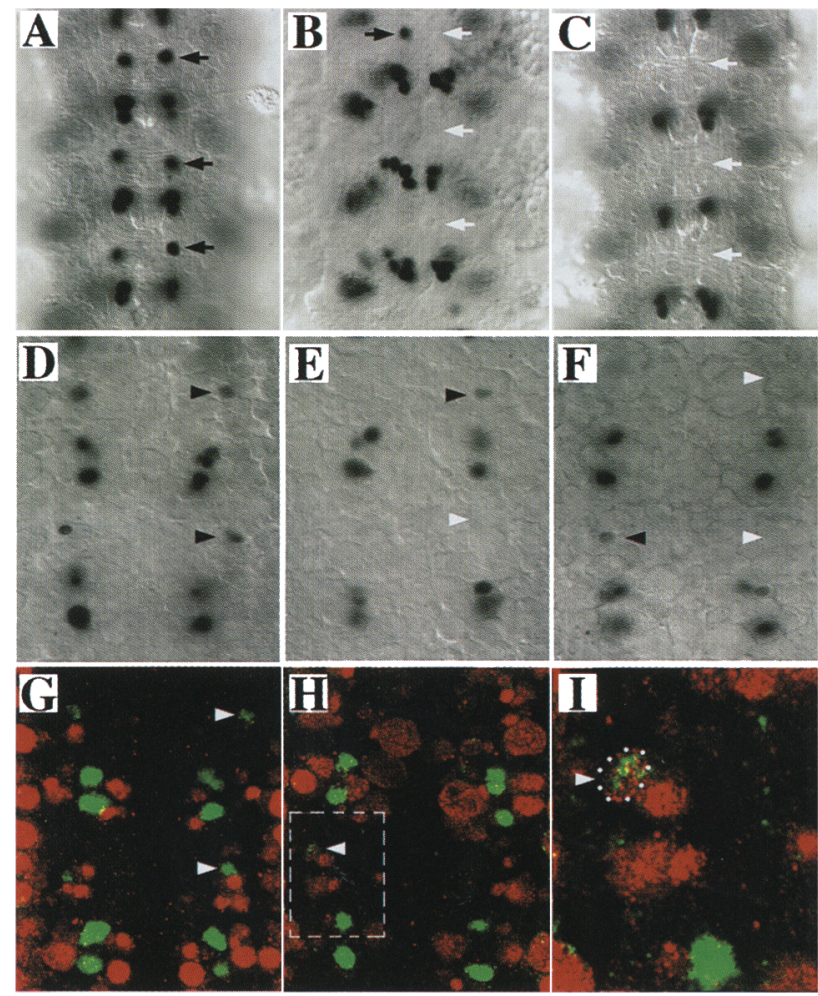

Figure 7. Ectopically expressed Klu protein inhibits formation of $\mathrm{Eve}^{+} \mathrm{GMC} 4-2 \mathrm{a}$ and mature RP2 neuron. $(A-C)$ Dorsal views of dissected stage- 15 embryos stained with anti-Eve to visualize mature RP2 neurons. (A) A control non-heat-shocked embryo homozygous for a HS70-klu insertion on the third chromosome showing the wild-type pattern of one Eve ${ }^{+}$RP2 neuron (black arrow) in every hemisegment. $|B|$ A heat-shocked embryo (see Materials and Methods) homozygous for the same third chromosomal HS70-klu insertion; note that for most hemisegments, an Eve ${ }^{+}$RP2 neuron is absent (white arrows indicate the positions of the absent RP2s); see text and Fig. 8 for quantitation. $(C)$ An embryo containing both the ftz-gal4 and the uasklu transgenes; note the absence of mature RP2 neurons in all hemisegments (see text). $(D-F)$ Ventral views of stage-11 embryos stained with anti-Eve to visualize GMC4-2a. (D) A con trol, non-heat-shocked embryo carrying the HS70-klu transgene showing the wild-type pattern of one Eve ${ }^{+}$GMC4-2a (black arrowhead) per hemisegment. $(E)$ A heat-shocked embryo carrying the $\mathrm{HS70-klu}$ transgene; note the absence (white arrowhead) of $\mathrm{Eve}^{+} \mathrm{GMC}^{-2}$ as in most of the hemisegments (see text). $(F)$ An embryo containing both the ftz-gal4 and the uas-klu transgenes; note the absence of $\mathrm{Eve}^{+} \mathrm{GMC4}-2$ as in most hemisegments (see text). (G-I) Superimposed confocal images of a antiEve (greenl and anti-Klu (red) double-labeled stage-11 embryo. Arrowhead indicates GMC4-2a. $(G)$ Wild-type embryo. $(H)$ Embryo containing both $f t z-g a l 4$ and uas-klu. (I) Enlarged image of GMC4-2a from $H$; note that this GMC4-2a expresses both Eve and Klu (yellow), albeit at low levels. Anterior is up.

ments fail to exhibit Eve ${ }^{+}$mature RP2 neurons (scored at stage 14 or later); whereas the non-heat-shocked controls (Fig. 7A) and heat-shocked wild-type embryos (data not shown) showed the wild-type anti-Eve staining pattern. The frequency of RP2 loss dropped drastically from 


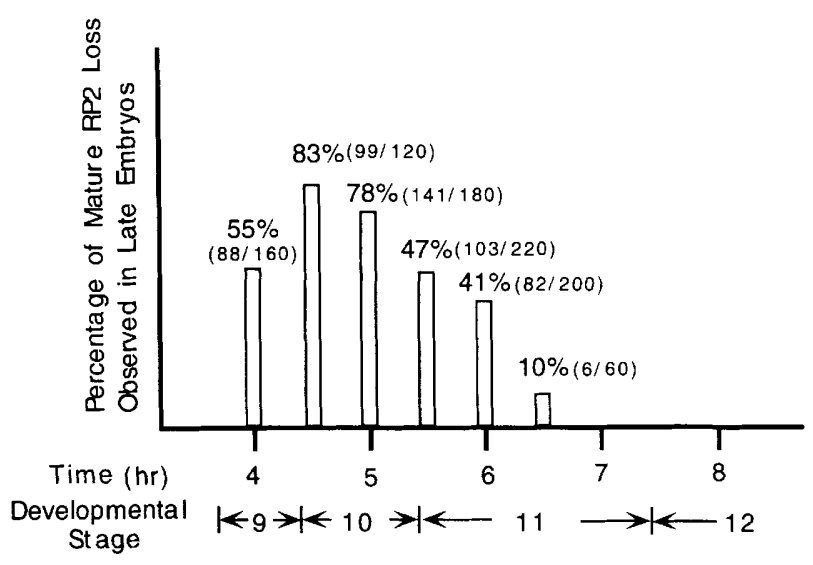

Figure 8. The temporal dependence of Klu ectopic expression in causing the loss of mature RP2 neurons. A 10-min heat shock was given at various stages of development to embryos homozygous for a third chromosome insertion containing a $H S P 70$ klu transgene; the embryos were aged until stage 15-16 and scored for the presence or absence of mature Eve ${ }^{+} \mathrm{RP} 2$ neurons (see Materials and Methods). Heat shock treatment at stage 10 results in the maximal loss of mature RP2 in late embryos. Number of hemisegments scored is given in parenthesis.

$\sim 80 \%$ to only $10 \%$ when the heat shock is administered 2 hr later at mid-stage 11 (Fig. 8). There is a temporal window within which ectopic Klu expression can prohibit formation of the mature RP2 neuron. This window coincides with the time frame of the birth of GMC4-2a in the developing embryonic CNS, suggesting that the ectopically expressed Klu might act at the GMC /GMC42a) level to prevent mature RP2 formation.

To directly examine this possibility, a similar heat shock experiment was carried out to look at the effects of ectopic Klu on the formation of Eve ${ }^{+}$GMC4-2a (scored at stage 11). Embryos heat-shocked at $4.5 \mathrm{hr}$ after egg-laying (early stage 10) lose Eve-expressing GMC4-2a (see Fig. 7E) from $77 \%(230 / 300)$ of their hemisegments. This is consistant with the frequency of RP2 loss in heatshocked embryos and lends further credence to the idea that ectopically expressed Klu can act to prevent GMC42a from being specified correctly, thereby preventing Eve-expressing RP2 neurons from being made. Both considerations of the timing of ectopic expression and the fact that the identity of GMC4-2a is affected argue against the defects being mediated at the level of the post-mitotic neurons. However, because of the crude nature of the heat shock paradigm, other possibilities cannot be rigorously ruled out; for example, it is conceivable that as the result of ectopically expressing Klu, NB4-2 might adopt a NB4-2b fate, thereby failing to produce the first two GMCs in the lineage, GMC4-2a and GMC4-2b, as well as their progeny.

Ectopically expressed Klu in GMC4-2a is sufficient to prevent the formation of Eve ${ }^{+}$GMC4-2a and mature RP2 neuron

To demonstrate more rigorously that ectopically ex- pressed Klu does act at the GMC4-2a level to prevent the formation of Eve-expressing RP2 neurons, experiments of targeted ectopic expression of klu in GMC4-2a were carried out with the Gal4 system (Brand and Perrimon 1993). We used a ftz-gal4 driver that is expressed in -20 GMCs, including GMC4-2a, but not in NBs. Animals homozygous for either the ftz-gal4 or uas-klu transgene alone did not show any abnormalities in the anti-Eve staining pattern (data not shown). In embryos carrying both the ftz-gal4 and uas-klu transgenes, $82 \%(214 / 260)$ of the hemisegments failed to exhibit an Eve RP2 neuron (Fig. 7C). Ectopic klu expression driven by the $f t z$ promotor can be detected by the anti-Klu antibody in GMC4-2a in stage 11 embryos (Fig. 7H,I), albeit at a low level. In embryos carrying both $\mathrm{ftz}-\mathrm{gal} 4$ and uas-klu transgenes, Eve expression in GMC4-2a was either lost completely or was weak (Fig. 7F,H,I). Seventy-nine percent $(283 / 360)$ of hemisegments failed to produce a Eve ${ }^{+}$ GMC4-2a at stage 11. This experiment demonstrates that ectopic expression of Klu in GMC4-2a is sufficient to prohibit the formation of Eve ${ }^{+}$GMC4-2a.

The possiblity that ectopically expressed Klu protein in NB4-2 might cause it to adopt an NB4-2b identity (thereby eliminating GMC4-2a and GMC4-2b and their respective progeny from the lineage) can be eliminated because the $f t z-g a l 4$ driver does not express in any NB. Based on these ectopic expression experiments, we conclude that ectopic expression of Klu in GMC4-2a is sufficient to alter the identity of GMC4-2a, leading ultimately to a decrease in the number of mature RP2 neurons. It is possible that ectopically expressed Klu in GMC4-2a forces it to adopt a GMC4-2b identity; however, because of the lack of information on the nature of the progeny of GMC4-2b, we cannot directly test this prediction.

\section{Discussion}

The role of $\mathrm{klu}$ within the context of genes affecting the NB4-2 lineage

A number of processes and mechanisms need to be studied in an effort to understand how cellular diversity is generated in the CNS-the establishment of the initial identity of the NBs; the asymmetric NB cell division; the modulation of NB identity with each cell cycle; and related to this issue, the establishment of distinct GMCs within each NB lineage; the GMC cell division and the signalling events that must occur at the level of the postmitotic neurons to establish asymmetric cell fate. To identify the molecules and elucidate the mechanisms involved in these processes and how they work with respect to a single NB lineage, the NB4-2 lineage, considerable attention has been focused on mutations that affect RP2 cell number.

To date, several genes have been identified that act at various levels to affect the genesis of this cell. At the NB level, Wg protein secreted by cells that are destined to form row $5 \mathrm{NBs}$ is required for the formation/specification of NB4-2 as well as other row 4 and row $6 \mathrm{NBs}$ 
(Chu-LaGraff and Doe 1993). In the absence or under conditions of limiting $w g$ function, correctly specified GMC4-2a and RP2 are not made. Two other segment polarity genes, patched (ptc) and gooseberry $(g s b)$, are also involved in controlling the $w g$-mediated specification of NB4-2 (Patel et al. 1989a; Zhang et al. 1994; Skeath et al. 1995; Bhat 1996). Although segment polarity genes like $w g, g s b$, and $p t c$ have been shown to act before NB delamination (for review, see Doe and Skeath 1996) to specify NB identity along the anterior-posterior axis, few genes have been described that act to differentiate between the identities of NBs along the dorsal/ventral axis. A gene, lottchen, appears to act to differentiate between the identities of NB4-3 and NB4-2; in lottchen loss-of-function mutants, NB4-3 apparently takes on some of the characteristics of NB4-2 (Buescher and Chia 1997).

The understanding of cell fate specification within NB lineages has been hampered by the lack of lineage informations and markers. The adaptation of the DiI labeling technique to Drosophila has facilitated the determination of terminal NB lineages; however information pertaining to the order in which these cells are generated and markers that facilitate the identification of these cells are largely lacking. Therefore, the analysis of cell fate specification within NB lineages has largely been limited to the first GMC and its progeny (see Introduction). Although it has been shown that gene expression changes in the course of an NB lineage (e.g., Cui and Doe 1992; Broadus et al. 1995), and it has been postulated that there must be genes that act to differentiate between cells born at different times within one stem cell lineage (Doe 1992), this point has not been demonstrated. In this study, we extend the analysis of the NB4-2 lineage to its second-born GMC. Our data show that a single genetic function encoded by klu is sufficient to differentiate between the second-born GMC4-2b from the first-born GMC4-2a. Klu is expressed in GMC4-2b but is absent in the earlier born cells in the lineage (NB4-2, NB4-2a, and GMC4-2a). Our results indicate that, consistent with its expression pattern, Klu acts at the GMC and not at the NB level. In the absence of Klu, GMC4-2b adopts a GMC4-2a identity, for example, expresses Eve and divides to produce progeny with characteristics normally associated with the progeny of GMC4-2a. Furthermore, the mis-expression of Klu in GMC4-2a prevents this cell from acquiring its correct cellular identity and prevents the formation of a mature RP2 neuron.

\section{When does Klu act to establish GMC4-2b identity?}

When is the GMC4-2b identity normally established? How and when does the mutant GMC4-2b adopt a GMC4-2a identity? The fact that the enhancer trap allele $\mathrm{klu}^{\mathrm{P} 1741}$ expresses $\beta$-Gal in phenotypically mutant GMC4-2b is interesting to consider; the fact that this cell initiates klu ( $\beta$-Gal) expression suggests that the mutant GMC4-2b is born with an initially normal GMC4$2 \mathrm{~b}$ identity (presumably because the cell identity of its mother NB4-2a, which does not express Klu, is not al- tered in the mutant); however, this identity is clearly not maintained in the mutant because this cell proceeds to acquire characteristics of GMC4-2a. So at the time of its birth, the mutant GMC4-2b clearly has a distinct identity from GMC4-2a and initiates klu ( $\beta$-Gal) expression. However, in the absence of the Klu function, this distinction from GMC4-2a cannot be maintained and this cell ultimately adopts a GMC4-2a identity.

These observations suggest that the initial identity of any GMC is specified by its mother NB. In this context, our observations lend support to the view that gene products present in the once divided NB, NB4-2a, are inherited by its daughter GMC, GMC4-2b; these gene products must act to initiate a program necessary to establish GMC4-2b cell identity. A key player that is normally activated in GMC4-2b to implement this program is Klu. Therefore, at least two types of genes are necessary to establish the identity of a GMC; first, genes that are expressed in the NB and presumably inherited by the GMC daughter cell which act to initiate a program necessary to establish GMC identity; second, genes encoding molecules like Klu that are expressed, presumably in response to the inherited gene products, and act exclusively in the GMC to establish its cellular identity.

Another point of interest is that, if this program for establishing GMC4-2b identity cannot be implemented attributable to the absence of or limiting klu function, GMC4-2b will adopt, as far as we can tell, all of the characteristics of GMC4-2a. GMC4-2b is clearly different from GMC4-2a early during its life because GMC4$2 \mathrm{~b}$ will initiate klu expression, whereas GMC4-2a does not. Nevertheless, GMC4-2b appears to retain a default program that will confer a GMC4-2a cell identity if the program (involving klu) necessary to establish a GMC4$2 \mathrm{~b}$ cell identity cannot be implemented, for example, in klu mutants. Therefore the absence or presence of a single gene product $(\mathrm{Klu})$ determines whether the second-born GMC in the NB4-2 lineage adopts a GMC4-2a or a GMC4-2b cell identity.

\section{Homeotic control}

The loss-of-function phenotype of klu appears to be highly position dependent. In klu protein null alleles, the RP2 duplication phenotype occurs with essentially complete expressivity $1>99 \%$ of the hemisegments exhibit RP2 duplications) in the more anterior segments, for example, T2-A1, whereas the expressivity in the more posterior segments (A2-A8) is much lower (see Results). The position dependence of the loss-of-function phenotype correlates well with the position-dependent expression of Klu in the CNS, where the levels of Klu protein expression appear to be much higher in the thoracic segments than in the abdominal segments (see Results). These observations suggest that the level of klu expression may be modulated by the homeotic genes.

There is precedence for homeotic regulation of the levels of gene expression in the CNS; previous findings further indicate that cell lineage regulation of insect CNS stem cells can be modified by homeotic genes (Taghert 
and Goodman 1984; Prokop and Technau 1994). The $p d m-1$ and $p d m-2$ genes that are required for the specification of GMC4-2a identity (Yeo et al. 1995) are expressed in the developing CNS with higher levels in the thoracic segments and lower levels in the abdominal seg. ments. The low level of expression in the abdominal segments appears to require genes of the bithorax complex (Lloyd and Sakonju 1991). A functional correlation to the positional dependence of the $p d m-1$ and $p d m-2$ expression levels has also been reported; when $p d m-2$ is ectopically expressed, duplications of the GMC4-2a sublineage occur apparently because both of the postmitotic progeny of GMC4-2a adopt the identity of their mother; this duplication appears to occur preferentially in the thoracic segments (Yang et al. 1993). The relatively low phenotypic penetrance seen specifically in the abdominal segments of klu null alleles suggests that in this region of the CNS where Klu expression is kept at a low level (presumably through the actions of homeotic genes), there are other players whose functions overlap those of klu.

\section{Materials and methods}

\section{Standard molecular biology techniques}

All routine DNA work was performed as described by Sambrook et al. (1989).

\section{Antibody preparation}

A fusion protein was produced using the QIAexpress system from QIAGEN. A 840-bp SphI (1995)/ Xhol (2835) fragment from the klu cDNA that encodes 280 amino acids (residues 433-712) from the carboxy-terminal region of $\mathrm{KLU}$ including the four $\mathrm{Zn}$ finger motifs was cloned in-frame with the $6 \mathrm{His}$ tag. Bacterially produced fusion protein was purified using Ni-NTA resin that is selective for proteins with an affinity tag of six consecutive histidine residues and injected into rabbits. Purified fusion protein $(300 \mu \mathrm{g})$ was used for the initial inoculation (complete Freund's adjuvant) and subsequent boosts (incomplete Freund's adjuvant). Anti-Klu serum was diluted 1:1000 and preadsorbed against fixed embryos before use. The authenticity of the wildtype staining patterns seen with the serum was confirmed by showing that embryos homozygous for $D f(3 R) k l l^{X R 19}$ or $\mathrm{klu}^{p 2121 R 51 \mathrm{C}}$ do not stain.

\section{Immunocytochemistry and photomicroscopy}

Single antibody staining was performed essentially as described by Yang et al. (1991). Embryos were collected, dechorionated with $50 \%$ bleach, fixed with $4 \%$ paraformaldehyde for $20 \mathrm{~min}$, devitellinized with a 1:1 mixture of heptane/methanol, washed with methanol, and rehydrated with PBT. Photomicrographs were taken with a Zeiss axiophot using DIC optics and either a $63 \times$ plan-Apochromat or a $20 \times$ Neofluor lens. For immunofluorescence, FITC- and Texas Red-conjugated anti-mouse or antirabbit antibodies (Jackson laboratory) were used. Lethal klu alleles were balanced over a "blue balancer" chromosome carrying a $u b x-l a c Z$ insertion and homozygous mutant embryos were identified by double staining with anti- $\beta$-Gal antibodies (Promega or Cappel) based on the absence of lacZ expression. Confocal microscopy was done on a Biorad MRC 600, using Adobe Photoshop 3.0 for image processing.

\section{Generation of $\beta$-gal-expressing "flip-out" clones}

To label individual NB lineages through the expression of Taußgal, we used the FRT/FLP system (Golic and Lindquist 1989; Xu and Rubin 1993). Using a homozygous viable second chromosome insertion carrying hSFLP and a second chromosome insertion carrying an actin-Taußgal cassette (A. MartiSubirana and R. Holmgren, pers. comm.l, the stocks hsFLP/ CyO; klu p212lR51C/TM3, and Taußgal cassette/CyO; klup212IR51C/ TM3 were established; virgins from the first stock were crossed to males of the second stock. The actin-Taußgal transgene is normally inactive because the ubiquitous actin5c promoter is separated from the Taußgal by a sequence, in the form of a FRT cassette, which prevents its expression; however, when the FRT cassette is deleted through the expression of FLP, Taußgal will be expressed from the actin $5 c$ promoter. To irreversibly activate the expression of Tau- $\beta g a l$, embryos were collected from the above cross in 2 -hr intervals and aged for $2 \mathrm{hr}$ at $25^{\circ} \mathrm{C}$. Heat shock was performed by placing embryos into a moist chamber that was submerged in a waterbath for $15-20 \mathrm{~min}$ at $34^{\circ} \mathrm{C}$. Subsequently, the embryos were allowed to develop at $18^{\circ} \mathrm{C}$ until stage 15-16, fixed and stained with anti- $\beta$-Gal [Cappel) and antiEve. Non-heat-shocked control embryos handled in the same manner had no $\beta$-Gal ${ }^{+}$clones.

\section{hsp70-klu and uas-klu constructs and transformants}

To generate the hsp 70-klu construct, full-length klu cDNA plasmid was linearized with $H$ indIII and rendered blunt-ended by end-filling with Klenow polymerase; an $\sim 4$-kb NotI/endfilled HindII fragment, containing the whole of the klu coding region was generated by digesting with NotI. This fragment was inserted into HpaI/NotI cut pCaSpeR-hs /from Carl Thummel, University of Utah, Salt Lake Cityl for germ-line transformation.

To produce a full-length klu cDNA under the control of gal4uas, an EcoRI site in the $5^{\prime}$ untranslated region (UTR) of klu was engineered by PCR. The $5^{\prime}$ primer (gaattcaagcttgaattccaataacgatcggcgcgt|contained an EcoRI site and sequence from the klu $5^{\prime}$-UTR. The $3^{\prime}$ internal klu primer (atcgctgcagatctggca) used in the PCR reaction includes the BgIII site at position 487. The 387-bp PCR product was cut with EcoRI and BgIII and added to a ligation mix that contained the gal4-uas germ-line transformation vector, pUAST, cut with EcoRI and the remaining 3351bp $B g I I I / E c o$ RI fragment from the klu cDNA. The final construct containing the 3738-bp klu cDNA was selected by restriction mapping.

The procedure described by Spradling (1986) was used to obtain germ-line transformants.

\section{klu misexpression experiments}

Heat shock-induced ectopic expression experiments were carried out with a homozygous transformant line that carried an HSP70-klu insertion on the third chromosome. Overnight collection of embryos were dechorionated; stage 7 embryos $2 \mathrm{hr} 50$ min to $3 \mathrm{hr}$ ) were hand-picked, transferred to a moist chamber and aged for various times at $25^{\circ} \mathrm{C}$ before being treated to a 10 -min heat shock at $37^{\circ} \mathrm{C}$. The heat-shocked embryos were kept in the moist chamber at $18^{\circ} \mathrm{C}$ and allowed to develop until stage 15-16; the embryos were fixed, stained with anti-Eve and $\mathrm{Eve}^{+} \mathrm{RP2}$ s were scored. A similar experiment was carried out to assess the formation of Eve ${ }^{+}$GMC-2a. Hand-picked stage-7 embryos were incubated in a moist chamber at $25^{\circ} \mathrm{C}$ for $1.5 \mathrm{hr}$. After a 10 -min heat shock at $37^{\circ} \mathrm{C}$ the embryos were aged for another $3.5 \mathrm{hr}$ at $25^{\circ} \mathrm{C}$. The embryos were fixed and stained 
with anti-Eve and Eve ${ }^{+}$GMC4-2as were scored. Control embryos comprising of heat-shocked wild-type embryos and nonheat-shocked HSP70-klu embryos were treated in a similar way.

For targeted misexpression of Klu, the $f t z-g a 14 / T M 3, S b$ Gal4 driver line (from the C.S. Goodman laboratory, homozygous lethal) was used to drive the expression of a uas-klu insertion on the second chromosome. A stock, uas-klu/Cyo; ftz-gal4/ $T M 3$, carrying both the $f t z-g a l 4$ and uas-klu-myc insertions was generated; overnight collections of embryos from this stock were used for the study. Control embryos homozygous for $f t z-$ gal4 or homozygous for uas-klu alone did not show any defects in RP2 formation.

\section{Staging embryos}

In general, we use the morphological criteria set out by CamposOrtega and Hartenstein (1985). The criteria we use for identifying NB4-2, NB4-2a, and GMC4-2a as well as the criteria we use for making fine distinctions of the age of embryos from stage 10-13 have been described previously (Yang et al. 1993).

\section{Acknowledgments}

We thank Jose Campos-Ortega for generously providing klu mutant alleles and all available sequence information and materials pertaining to klu before publication; A. Marti-Subirana and R. Holmgren for providing transformants of the tau-lac $Z$ cassette before publication; C.Q. Doe, M. Frasch, W. Gehring, C.S. Goodman, Z. Lai, and K. Zinn for providing antibodies and/or flies; Kathy Matthews and the Bloomington stock center for efficiently providing stocks. We also thank Nick Harden, Gerald Udolph, and members of the Chia laboratory for helpful discussions and comments on the manuscript. This work is supported by the National Science and Technology Board and the European Community.

The publication costs of this article were defrayed in part by payment of page charges. This article must therefore be hereby marked "advertisement" in accordance with 18 USC section 1734 solely to indicate this fact.

\section{References}

Bhat, K. 1996. The patched signaling pathway mediates repression of gooseberry allowing neuroblast specification by wingless during Drosophila neurogenesis. Development 122: 2921-2932.

Bhat, K., S. Poole, and P. Schedl. 1995. The miti-mere and pdm-1 genes collaborate during specification of the RP2/ sibling lineage in Drosophila neurogenesis. Mol. Cell. Biol. 15: 4052-4063.

Billin, A.N., K.A. Cockerill, and S.J. Poole. 1991. Isolation of a family of Drosophila POU domain genes expressed in early development. Mech. Dev. 34: 75-84.

Bossing, T. and G. Technau. 1994. The fate of midline progenitors in Drosophila as revealed by a new method for single cell labelling. Development 120: 1895-1906.

Bossing, T., G. Technau, and C.Q. Doe. 1996a. huckebein is required for glial development and axon pathfinding in the neuroblast 1-1 and neuroblast 2-2 lineages in the Drosophila central nervous system. Mech. Dev. 55: 53-64.

Bossing, T., G. Udolph, C.Q. Doe, and G. Technau. 1996b. The embryonic CNS lineages of Drosophila melanogaster: I. Neuroblast lineages derived from the ventral half of the neuroectoderm. Dev. Biol. 179: 41-64.
Brand, A. and N. Perrimon. 1993. Targeted gene expression as a means of altering cell fates and generating dominant phenotypes. Development 118: 401-415.

Broadus, J., J.B. Skeath, E.P. Spana, T. Bossing, G. Technau, and C.Q. Doe. 1995. New neuroblast markers and the origin of the aCC and pCC neurons in the Drosophila central nervous system. Mech. Dev. 53: 393-402.

Buescher, M. and W. Chia. 1997. Mutations in lottchen cause cell fate transformations in both neuroblast and glioblast lineages in the Drosophila embryonic central nervous system. Development 124: 673-681.

Campos-Ortega, J.A. 1993. Early neurogenesis in Drosophila melanogaster. In The development of Drosophila melanogaster (ed. M. Bate and A. Martinez-Arias), pp. 1091-1131. Cold Spring Harbor Laboratory Press, Cold Spring Harbor, NY.

Campos-Ortega, J.A. and V. Hartenstein. 1985. The embryonic development of Drosophila melanogaster. Springer Verlag, Berlin, Germany.

Cui, X. and C.Q. Doe. 1992. ming is expressed in neuroblast sublineages and regulates gene expression in the Drosophila central nervous system. Development 116: 943-952.

Chu-LaGraff, Q. and C.Q. Doe. 1993. Neuroblast specification and formation regulated by wingless in the Drosophila CNS. Science 261: 1594-1597.

Chu-LaGraff, Q., A. Schmid, J. Leidel, G. Broenner, H. Jaeckle, and C.Q. Doe. 1995. huckebein specifies aspects of CNS precursor identity required for motoneuron axon pathfinding. Neuron 15: 1041-1051.

Dick, T., X. Yang, S. Yeo, and W. Chia. 1991. Two closely linked Drosophila POU domain genes are expressed in neuroblasts and sensory elements. Proc. Natl. Acad. Sci. 88: 7645-7649.

Doe, C.Q. 1992. Molecular markers for identified neuroblasts and ganglion mother cell in the Drosophila embryonic central nervous system. Development 116: 855-863.

Doe, C.Q. and G. Technau. 1993. Identification and cell lineage of individual neuroblasts in the Drosophila CNS. Trends Neurosci. 16: 510-514.

Doe, C.Q. and J.B. Skeath. 1996. Neurogenesis in the insect nervous system. Curr. Opin. Neurobiol. 6: 18-24.

Doe, C.Q., Y. Hiromi, W.J. Gehring, and C.S. Goodman. 1988a. Expression and function of the segemtation gene fushi tarazu during Drosophila neurogenesis. Science 239: 170175.

Doe, C.Q., D. Smouse, and C.S. Goodman. 1988b. Control of neuronal fate by the Drosophila segmentation gene evenskipped. Nature 333: 376-378.

Doe, C.Q., Q. Chu-LaGraff, D.M. Wright, and M.P. Scott. 1991. The prospero gene specifies cell fate in the Drosophila central nervous system. Cell 65: 451-465.

Frasch, M., T. Hoey, C. Rushlow, H. Doyle, and M. Levine. 1986. Characterisation and localisation of the even-skipped protein of Drosophila. EMBO I. 6: 749-759.

Fujita, S.C., S.L. Zipursky, S. Benzer, A. Ferrus, and S.L. Shotwell. 1982. Monoclonal antibodies against the Drosophila nervous system. Proc. Natl. Acad. Sci. 79: 7929-7933.

Golic, K.G. and S. Lindquist. 1989. The FLP recombinase of yeast catalyses site-specific recombination in the Drosophila genome. Cell 59: 499-509.

Goodman, C.S and C.Q. Doe. 1993. Embryonic development of the Drosophila central nervous system. In The development of Drosophila melanogaster (ed. M. Bate and A. MartinezArias), pp. 1131-1206. Cold Spring Harbor Laboratory Press, Cold Spring Harbor, NY.

Hartenstein, V. and J.A. Campos-Ortega. 1984. Early neurogen- 
esis in wildtype Drosophila melanogaster. Wilhelm Roux's Arch. Dev. Biol. 193: 308-325.

Hirata, J., H. Nakagoshi, Y. Nabeshima, and F. Matsuzaki. 1995. Asymmetric segregation of a homeoprotein, prospero, during cell division in neural and endodermal development. Nature 377: 627-630.

Ito, K., J. Urban, and G.M. Technau. 1995. Distribution, classification and development of Drosophila glial cells in the late embryonic and early larval ventral nerve cord. Wilhelm Roux's Arch. Dev. Biol. 204: 284-307.

Jimenez, F. and J. Modolell. 1993. Neural fate specification in Drosophila. Curr. Opin. Genet. Dev. 3: 626-632.

Klein, T. and J.A. Campos-Ortega 1997. klumpfuss, a Drosophila gene similar to the Wilms' tumor suppressor gene, is involved in bristle and leg development. Development (in press).

Knoblich, J.A., L.Y. Jan, and Y.N. Jan. 1995. Localisation of numb and prospero reveals a novel mechanism for asymmetric protein segregation during mitosis. Nature 377: 624-627.

Lai, Z., M.E. Fortini, and G.M. Rubin. 1991. The embryonic expression pattern of $z f h-1$ and $z f h-2$, two Drosophila genes encoding novel zinc-finger homeodomain proteins. Mech. Dev. 34: 123-134.

Lloyd, A. and S. Sakonju. 1991. Characterization of two Drosophila POU domain genes, related to oct-1 and oct-2, and the regulation of their expression pattern. Mech. Dev. 36: 87-102.

Matsuzaki, F., K. Koizumi, C. Hama, T. Yoshioka, and Y. Nabeshima. 1991. Cloning of the Drosophila prospero gene and its expression in ganglion mother cells. Biochem. Biophys. Res. Comm. 182: 1326-1332.

Patel, N.H., B. Schafer, C.S. Goodman, and R. Holmgren. 1989a. The role of segment polarity genes during Drosophila neurogenesis. Genes \& Dev. 3: 890-904.

Patel, N.H., E. Martin-Blanco, K.G. Coleman, S.J. Poole, M.C. Ellis, T.B. Kornberg, and C.S. Goodman. 1989b. Expression of engrailed proteins in arthropods, annelids and chordates. Cell 58: 955-968.

Prokop, A. and G.M. Technau. 1994. Early tagma-specific commitment of Drosophila CNS progenitor NB1-1. Development 120: 2567-2578.

Sambrook, J., E. Frisch, and T. Maniatis. 1989. Molecular cloning: A laboratory manual. Cold Spring Harbor Laboratory Press, Cold Spring Harbor, NY.

Skeath, J.B., Y. Zhang, R. Holmgren, S.B. Carrol, and C.Q. Doe. 1995. Specification of neuroblast identity in the Drosophila embryonic central nervous system by gooseberry-distal. $\mathrm{Na}$. ture 376: 427-430.

Spana, E. and C.Q. Doe. 1995. The prospero transcription factor is asymmetrically localised to the cell cortex during neuroblast mitosis in Drosophila. Development 121: 3187-3195.

Spradling, A.C. 1986. P-element mediated transformation. In Drosophila: A practical approach (ed. D.B. Roberts), pp. 175197. IRL Press, Oxford, England.

Taghert, P. and C.S. Goodman. 1984. Cell determination and differentiation of identified serotonin-immunoreactive neurons in the grasshopper embryo. I. Neurosci. 4: 989-1000.

Udolph, G., A. Prokop, T. Bossing, and G.M. Technau. 1993. A common precursor for glia and neurons in the embryonic CNS of Drosophila gives rise to segment specific lineage variants. Development 118: 765-775.

Udolph, G., K. Lüer, T. Bossing, and G.M. Technau. 1995. Commitment of CNS progenitors along the dorsoventral axis of Drosophila neuroectoderm. Science 269: 1278-1281.

Vaessin, H., E. Greel, E. Wolff, E. Bier, L.Y. Jan, and Y.N. Jan. 1991. prospero is expressed in neuronal precursors and en- codes a nuclear protein that is involved in the control of axonal outgrowth in Drosophila. Cell 67: 941-953.

Van Vactor, D., H. Sink, D. Fambrough, R. Tsoo, and C.S. Goodman. 1993. Genes that control neuromuscular specificity in Drosophila. Cell 73: 1137-1153.

Xu, T. and G. Rubin. 1993. Analysis of genetic mosaics in developing and adult Drosophila tissues. Development 117: 1223-1237.

Yang, X., K. Seow, S. Bahri, S. Oon, and W. Chia. 1991. Two Drosophila receptor-like tyrosine phosphatase genes are specifically expressed in a subset of developing axons and pioneer neurons in the embryonic CNS. Cell 67: 661-673.

Yang, X., S. Yeo, T. Dick, and W. Chia. 1993. The role of a Drosophila POU homeodomain gene in the specification of neural precursor cell identity in the developing embryonic central nervous system. Genes \& Dev. 7: 504-516.

Yeo, S., A. Lloyd, K. Kozak, A. Dinh, T. Dick, X. Yang, S. Sakonju, and W. Chia. 1995. On the functional overlap between two Drosophila POU homeodomain genes and the cell fate specification of a CNS neural precursor. Genes \& Dev. 9: 1223-1236.

Zhang, Y., A. Ungar, C. Fresquez, and R. Holmgren. 1994. Ectopic expression of either gooseberry-distal or proximal gene causes alterations of cell fate in the epidermis and central nervous system. Development 120: 1151-1161. 


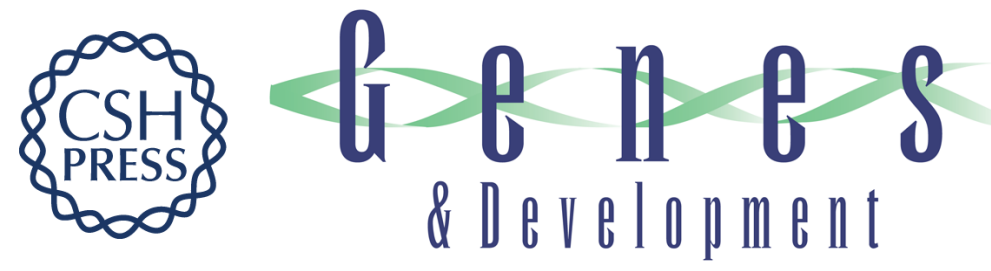

\section{Klumpfuss, a putative Drosophila zinc finger transcription factor, acts to differentiate between the identities of two secondary precursor cells within one neuroblast lineage.}

$X$ Yang, S Bahri, T Klein, et al.

Genes Dev. 1997, 11:

Access the most recent version at doi:10.1101/gad.11.11.1396

References This article cites 45 articles, 21 of which can be accessed free at:

http://genesdev.cshlp.org/content/11/11/1396.full.html\#ref-list-1

License

Email Alerting Service

Receive free email alerts when new articles cite this article - sign up in the box at the top right corner of the article or click here.

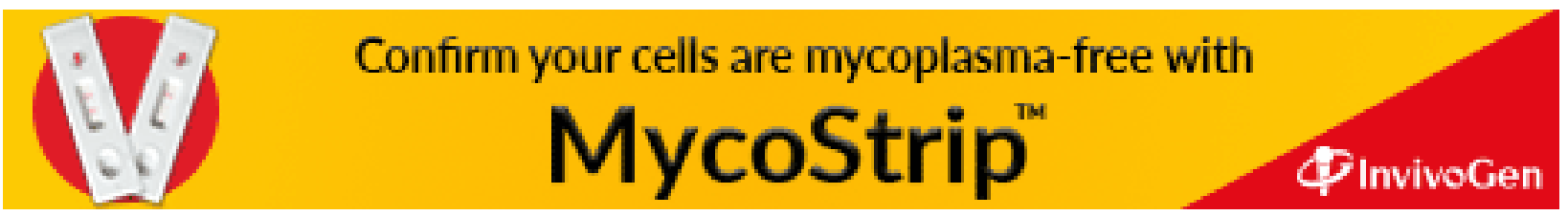

